\title{
ARES. III. Unveiling the Two Faces of KELT-7 b with HST WFC3*
}

\author{
William Pluriel $^{1}$, Niall Whiteford ${ }^{2,3}$, Billy Edwards ${ }^{4}$ (i), Quentin Changeat ${ }^{4}$ (1), Kai Hou Yip ${ }^{4}$, Robin Baeyens ${ }^{5}$, \\ Ahmed Al-Refaie ${ }^{4}$ (1), Michelle Fabienne Bieger ${ }^{6}$, Dorian Blain ${ }^{7}$, Amélie Gressier ${ }^{7,8}$, Gloria Guilluy ${ }^{9,10}$, Adam Yassin Jaziri ${ }^{1}$, \\ Flavien Kiefer ${ }^{8}$, Darius Modirrousta-Galian ${ }^{11,12}$, Mario Morvan ${ }^{4}$ (1) , Lorenzo V. Mugnai ${ }^{13}$ (1), Mathilde Poveda ${ }^{14,15}$, Nour Skaf ${ }^{4,7}$ (D),

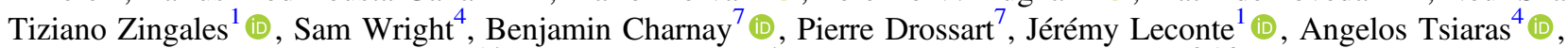 \\ Olivia Venot ${ }^{14}$ (1) Ingo Waldmann $^{4}$ (i), and Jean-Philippe Beaulieu ${ }^{8,16}$ (1) \\ ${ }^{1}$ Laboratoire d'astrophysique de Bordeaux, Univ. Bordeaux, CNRS, B18N, allée Geoffroy Saint-Hilaire, F-33615 Pessac, France; william.pluriel@u-bordeaux.fr \\ ${ }^{2}$ Institute for Astronomy, University of Edinburgh, Blackford Hill, Edinburgh, EH9 3HJ, UK \\ ${ }^{3}$ Centre for Exoplanet Science, University of Edinburgh, Edinburgh, EH9 3FD, UK \\ ${ }^{4}$ Department of Physics and Astronomy, University College London, London, UK \\ ${ }^{5}$ Instituut voor Sterrenkunde, KU Leuven, Celestijnenlaan 200D bus 2401, B-3001 Leuven, Belgium \\ ${ }^{6}$ College of Engineering, Mathematics and Physical Sciences, Physics Building, University of Exeter, North Park Road, Exeter, UK \\ ${ }^{7}$ LESIA, Observatoire de Paris, Université PSL, CNRS, Sorbonne Université, Université de Paris, 5 place Jules Janssen, F-92195 Meudon, France \\ ${ }^{8}$ Sorbonne Universités, UPMC Université Paris 6 et CNRS, UMR 7095, Institut d'Astrophysique de Paris, 98 bis bd Arago, F-75014 Paris, France \\ ${ }^{9}$ Dipartimento di Fisica, Universitá degli Studi di Torino, via Pietro Giuria 1, I-10125 Torino, Italy \\ ${ }^{10}$ INAF Osservatorio Astrofisico di Torino, Via Osservatorio 20, I-10025 Pino Torinese, Italy \\ ${ }^{11}$ INAF-Osservatorio Astronomico di Palermo, Piazza del Parlamento 1, I-90134 Palermo, Italy \\ ${ }^{12}$ University of Palermo, Department of Physics and Chemistry, Via Archirafi 36, Palermo, Italy \\ ${ }^{13}$ La Sapienza Universitá di Roma, Department of Physics, Piazzale Aldo Moro 2, I-00185 Roma, Italy \\ ${ }^{14}$ Laboratoire Interuniversitaire des Systèmes Atmosphériques (LISA), UMR CNRS 7583, Université Paris-Est-Créteil, Université de Paris, Institut Pierre Simon \\ Laplace, Créteil, France \\ ${ }^{15}$ Maison de la Simulation, CEA, CNRS, Univ. Paris-Sud, UVSQ, Université Paris-Saclay, F-91191 Gif-sur-Yvette, France \\ ${ }^{16}$ School of Physical Sciences, University of Tasmania, Private Bag 37 Hobart, Tasmania 7001, Australia \\ Received 2020 April 1; revised 2020 May 31; accepted 2020 June 23; published 2020 August 14
}

\begin{abstract}
We present the analysis of the hot-Jupiter KELT-7 b using transmission and emission spectroscopy from the Hubble Space Telescope, both taken with the Wide Field Camera 3. Our study uncovers a rich transmission spectrum that is consistent with a cloud-free atmosphere and suggests the presence of $\mathrm{H}_{2} \mathrm{O}$ and $\mathrm{H}^{-}$. In contrast, the extracted emission spectrum does not contain strong absorption features and, although it is not consistent with a simple blackbody, it can be explained by a varying temperature-pressure profile, collision induced absorption, and $\mathrm{H}^{-}$. KELT-7 b had also been studied with other space-based instruments and we explore the effects of introducing these additional data sets. Further observations with Hubble, or the next generation of space-based telescopes, are needed to allow for the optical opacity source in transmission to be confirmed and for molecular features to be disentangled in emission.
\end{abstract}

Unified Astronomy Thesaurus concepts: Transmission spectroscopy (2133); Exoplanet atmospheres (487); Astronomy data analysis (1858)

\section{Introduction}

With thousands of planets detected during the previous two decades, the study of atmospheres is at the forefront of exoplanet research and spectroscopic observations now probe these worlds in search of molecular features. Such studies are crucial in the pursuit of understanding the diverse nature of exoplanet chemical compositions, atmospheric processes, internal structures, and the conditions required for planetary formation.

In recent years, there has been a surge in transit spectroscopy observations using both space-borne and ground-based facilities, resulting in significant advancements in our understanding of exoplanet atmospheres. This technique has been used for the detection of multiple molecular absorption features, including water $\left(\mathrm{H}_{2} \mathrm{O}\right.$; Tinetti et al. 2007; Tsiaras et al. 2019), methane $\left(\mathrm{CH}_{4}\right.$; Swain et al. 2008), and ammonia (MacDonald \& Madhusudhan 2017), becoming a cornerstone technique in the pursuit of exoplanet characterization. In particular, the Hubble Space Telescope (HST) has been widely used allowing us to characterize the atmospheres of several hot Jupiters (e.g., Deming et al. 2013; Wakeford et al. 2013; Haynes et al. 2015;

\footnotetext{
* ARES: Ariel Retrieval of Exoplanets School.
}

Kreidberg et al. 2018; Mikal-Evans et al. 2019) and has begun to observe enough planets for population studies to be undertaken (e.g., Sing et al. 2016; Tsiaras et al. 2018).

For hotter planets, HST transmission spectroscopy has provided evidence for absorption at shorter wavelengths. These are generally attributed to the presence of optical absorbers such as titanium oxide (TiO), vanadium oxide (VO), or iron hydride $(\mathrm{FeH})$. These planets include WASP-121b (MikalEvans et al. 2019) and WASP-127 b (Skaf et al. 2020). Additionally, high-resolution ground-based observations have detected the presence of a variety of heavy metals in the atmosphere of KELT-9 b (Hoeijmakers et al. 2019) while lower resolution data were used to claim the presence of aluminum oxide (AlO) in WASP-33 b (von Essen et al. 2019). Thermal emission spectra can also be used to characterize the dayside of exoplanets. In particular, hot Jupiters that emit significant and detectable infrared radiation are the ideal candidates for this approach. As thermal emission is sensitive to the temperaturepressure profile (Madhusudhan et al. 2014), it is possible to probe this structure and demonstrate how it can be driven by the presence of $\mathrm{TiO}$ in the atmosphere such as in the cases of WASP-33 b (Haynes et al. 2015) and WASP-76 b (Edwards et al. 2020a). 
Table 1

Target Parameters Used in This Study

\begin{tabular}{ll}
\hline \hline \multicolumn{2}{c}{ Stellar Parameters } \\
\hline Spectral type & $\mathrm{F}$ \\
Effective temperature $(\mathrm{K})$ & $6789_{-49}^{+50}$ \\
{$[$ Fe $/ \mathrm{H}]$} & $0.139_{-0.081}^{+.075}$ \\
Surface gravity (cgs) & $4.149_{-0.019}^{+0.019}$ \\
Radius $\left(R_{\odot}\right)$ & $1.732_{-0.045}^{+0.043}$ \\
\hline \multicolumn{2}{c}{ Planetary Parameters } \\
\hline Period (days) & $2.7347749_{-0.0000039}^{+0.0000039}$ \\
Inclination $($ deg $)$ & $83.76_{-0.37}^{+0.38}$ \\
Mass $\left(M_{\mathrm{J}}\right)$ & $1.28_{-0.18}^{+0.18}$ \\
Radius $\left(R_{\mathrm{J}}\right)$ & $1.533_{-0.047}^{+0.046}$ \\
Equilibrium temperature $(\mathrm{K})$ & $2048_{-27}^{+27}$ \\
$T_{0}\left(\mathrm{BJD} \mathrm{TBD}_{\mathrm{TB}}\right)$ & $2456355.229809_{-0.000198}^{+0.000198}$ \\
$a / R_{*}$ & $5.49_{-0.11}^{+0.12}$ \\
\hline Reference & Bieryla et al. $(2015)$ \\
\hline
\end{tabular}

The presence of these optical absorbers are an important component in current exoplanet atmospheric models, and are predicted to have a significant impact on the overall physics and chemistry of highly irradiated close-in giant planet atmospheres. Work by Fortney et al. (2008) suggests the dayside atmospheres of such planets can be divided into two separate classes. The first class represents atmospheres that are modeled to have significant opacity due to the presence of $\mathrm{TiO}$ and $\mathrm{VO}$ gases and thus results in, for example, atmospheric temperature inversions (with hot stratospheres). In the second case, atmospheres that do not possess opacity due to $\mathrm{TiO}$ and $\mathrm{VO}$ are modeled to redistribute absorbed energy more readily resulting in cooler daysides and warmer nightsides.

WASP-103 b, a similar planet of KELT-7 b, has been studied in both emission and transmission by HST, suggesting a thermal inversion on the dayside but with a featureless transmission spectrum (Kreidberg et al. 2018). On the other hand, HST data for WASP-76 b, another ultra-hot Jupiter, also showed a dayside thermal inversion due to the presence of $\mathrm{TiO}$ but a larger water feature and no evidence for optical absorbers in the transmission spectrum (Edwards et al. 2020a).

KELT-7 $\mathrm{b}$ is a transiting hot Jupiter, with a mass of $1.28_{-0.18}^{+0.18}$ $M_{\mathrm{J}}$, a radius of $1.533_{-0.047}^{+0.046} R_{\mathrm{J}}$, an orbital period of $2.7347749 \pm$ 0.0000039 days, and an equilibrium temperature of $\sim 2048 \mathrm{~K}$ (Bieryla et al. 2015; see Table 1). KELT-7 b, with relatively low surface gravity, high equilibrium temperature, and a bright host star, is an excellent candidate for thorough atmospheric characterization. It has previously been studied in both transmission and emission using the Spitzer Space Telescope's InfraRed Array Camera (IRAC; Garhart et al. 2020) and a ground-based eclipse was measured by Martioli et al. (2018). Here we present an analysis of this exoplanet using transmission and emission spectroscopy from the HST's Wide Field Camera 3 (WFC3). These HST observations allow for two complementary insights into the nature of this planet. We also explore the effects of combining the HST data set with those from Spitzer IRAC and the Transiting Exoplanet Survey Satellite (TESS).

\section{Data Analysis}

\subsection{Observational Data-HST}

We obtained the raw spectroscopic observation data from the Mikulski Archive for Space Telescopes (MAST). ${ }^{17}$ Both observations of KELT-7 b were undertaken in 2017 as part of Hubble proposal 14767 led by David Sing. These images are the result of a two visits of the target, each containing five HST orbits, using the infrared detector, the WFC3 G141 grism, and a scan rate of $0.9 \mathrm{~s}^{-1}$. Each image consists of five nondestructive reads with an aperture size of $266 \times 266$ pixels in the SPARS10 mode, resulting in a total exposure time of $22.317 \mathrm{~s}$, a maximum signal level of $33,000 \mathrm{e}^{-}$per pixel, and a total scan length of about 25."605. The spectral data used in this study are listed in the Table 4.

\subsection{Reduction and Extraction-Iraclis}

The data reduction and calibration was performed using the open-source software Iraclis (Tsiaras et al. 2016b). Our Iraclis analysis starts with the raw spatially scanned spectroscopic images. The data reduction and correction steps are performed in the following order: zero-read subtraction, reference pixel correction, nonlinearity correction, dark current subtraction, gain conversion, sky background subtraction, calibration, flat-field correction, bad pixels, and cosmic ray correction. A detailed description of the data reduction process could be found in Section 2 of Tsiaras et al. (2016b).

Following the reduction process, the flux was extracted from the spatially scanned spectroscopic images to create the final transit light curves per wavelength band. We considered one broadband (white) light curve covering the whole wavelength range in which the G141 grism is sensitive $(1.088-1.68 \mu \mathrm{m})$ and spectral light curves with a resolving power of 70 at $1.4 \mu \mathrm{m}$. The bands of the spectral light curves are selected such that the signal-to-noise ratio $(\mathrm{S} / \mathrm{N})$ is approximately uniform across the planetary spectrum. We extracted our final light curves from the differential, nondestructive reads. Prior to light curve fitting, we choose to discard the first HST orbit of each visit, as these exhibit much stronger hooks than subsequent orbits (Deming et al. 2013; Zhou et al. 2017).

Our white and spectral light curves were fit using the literature values from Table 2, with only two free parameters: the planet-to-star radius ratio and the mid-transit time. This is motivated by the Earth obscuration gaps, which often means that the ingress and egress of the transit are missed, limiting our ability to refine the semimajor-axisto-star radius ratio, the inclination, and the eccentricity. The limb-darkening coefficients were selected from the quadratic formula by Claret (2000), and using the stellar parameters in Table 1 and the ATLAS stellar models (Kurucz 1970; Espinoza \& Jordán 2015; Morello et al. 2020). Figure 1 shows the raw white light curve, the detrended white light curve, and the fitting residuals for both observations while Figure 2 shows the fits of spectral light curves for each wavelength.

\footnotetext{
${ }^{17}$ https://archive.stsci.edu/
} 

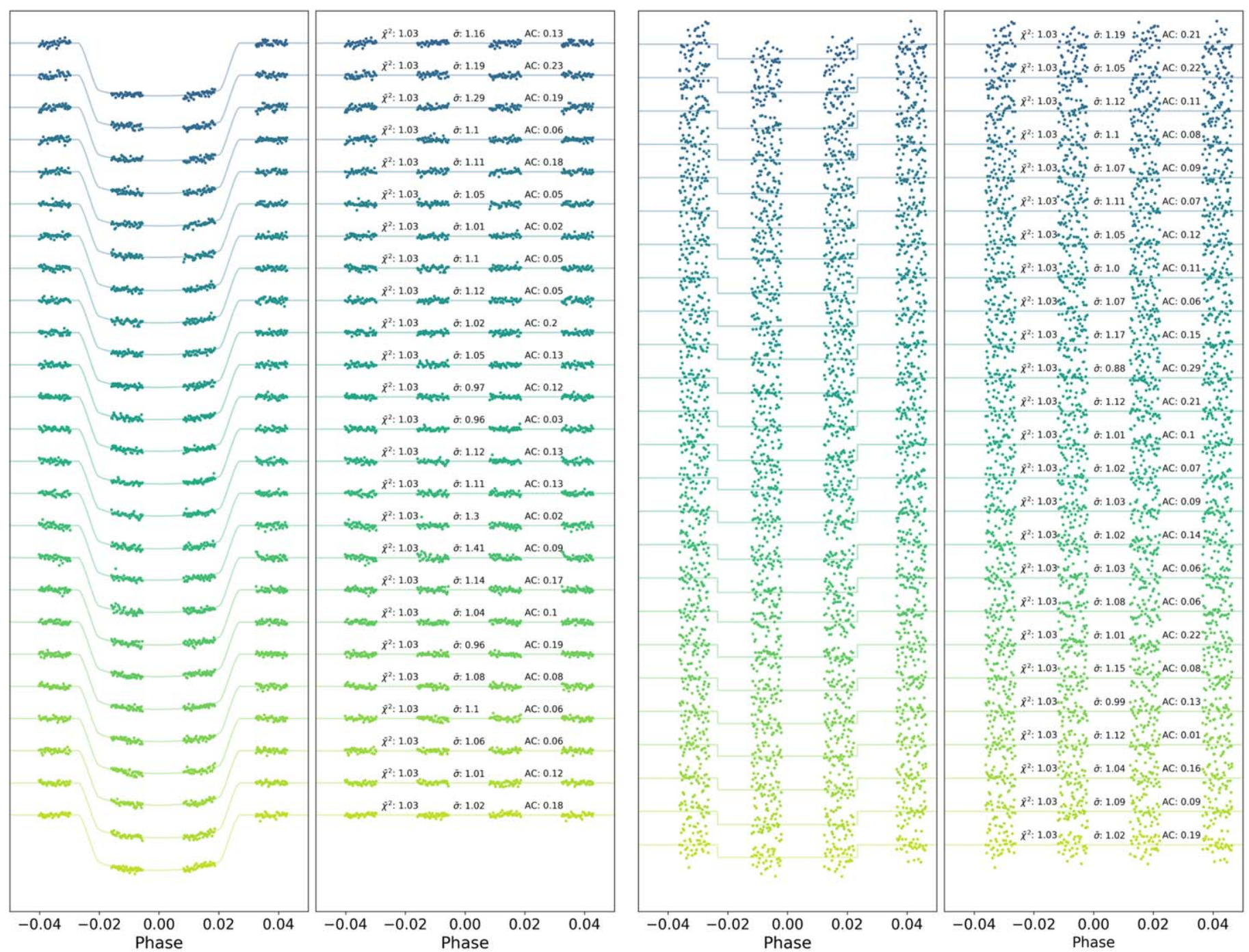

Figure 2. Spectral light-curve fits from Iraclis of transmission (left) and emission (bright) spectra—for clarity, an offset has been applied. In each plot, the left segment shows the spectral light curves while the residuals are shown in the right section. $\bar{\chi}$ is the reduced chi squared, $\bar{\sigma}$ is the ratio between the standard deviation of the residuals and the photon noise, and $\mathrm{AC}$ is the autocorrelation of the fitting residuals.

\subsection{Modeling Equilibrium Chemistry—petitCODE}

To help contextualize our free retrieval results, we computed a self-consistent forward model with petitCODE, a 1D pressure-temperature iterator solving for radiative-convective and chemical equilibrium (Mollière et al. 2015, 2017). The code includes opacities for $\mathrm{H}_{2}, \mathrm{H}^{-}, \mathrm{H}_{2} \mathrm{O}, \mathrm{CO}, \mathrm{CO}_{2}, \mathrm{CH}_{4}, \mathrm{HCN}$, $\mathrm{H}_{2} \mathrm{~S}, \mathrm{NH}_{3}, \mathrm{OH}, \mathrm{C}_{2} \mathrm{H}_{2}, \mathrm{PH}_{3}, \mathrm{SiO}, \mathrm{FeH}, \mathrm{Na}, \mathrm{K}, \mathrm{Fe}, \mathrm{Fe}^{+}, \mathrm{Mg}$, $\mathrm{Mg}^{+}, \mathrm{TiO}$, and $\mathrm{VO}$, as well as radiative scattering and CIA by $\mathrm{H}_{2}-\mathrm{H}_{2}$ and $\mathrm{H}_{2}-\mathrm{He}$. The atmosphere computed with petitCODE is assumed to be cloud-free, but the possibility of condensing refractory species is included in the equilibrium chemistry. Our petitCODE model for KELT-7 $b$ was computed using the stellar and planetary parameters determined by Bieryla et al. (2015). Here, the surface gravity was computed using the planetary mass and radius. Furthermore, an intrinsic temperature of $600 \mathrm{~K}$ was adopted, in accordance with its high equilibrium temperature (Thorngren et al. 2019). Finally, a global planetary averaged redistribution of the irradiation was assumed.

\subsection{Ephemeris Refinement}

Accurate knowledge of exoplanet transit times is fundamental for atmospheric studies. To ensure KELT-7 b can be observed in the future, we used our HST white light curve midtime, along with data from TESS (Ricker et al. 2014), to update the ephemeris of the planet. TESS data is publicly available through the MAST archive and we use the pipeline from Edwards et al. (2020b) to download, clean, and fit the twominute cadence data. KELT-7 b had been studied in Sector 19 and after excluding bad data, we recovered nine transits. These were fitted individually with the planet-to-star radius ratio $\left(R_{p} / R_{s}\right)$ and transit mid-time $\left(T_{\text {mid }}\right)$ as free parameters. We note that the ephemeris of KELT-7 b was also recently refined by Garhart et al. (2020) and we also used the mid-times derived in that study.

\section{Results}

Our analysis uncovers a rich transmission spectrum that is consistent with a cloud-free atmosphere and suggests the 


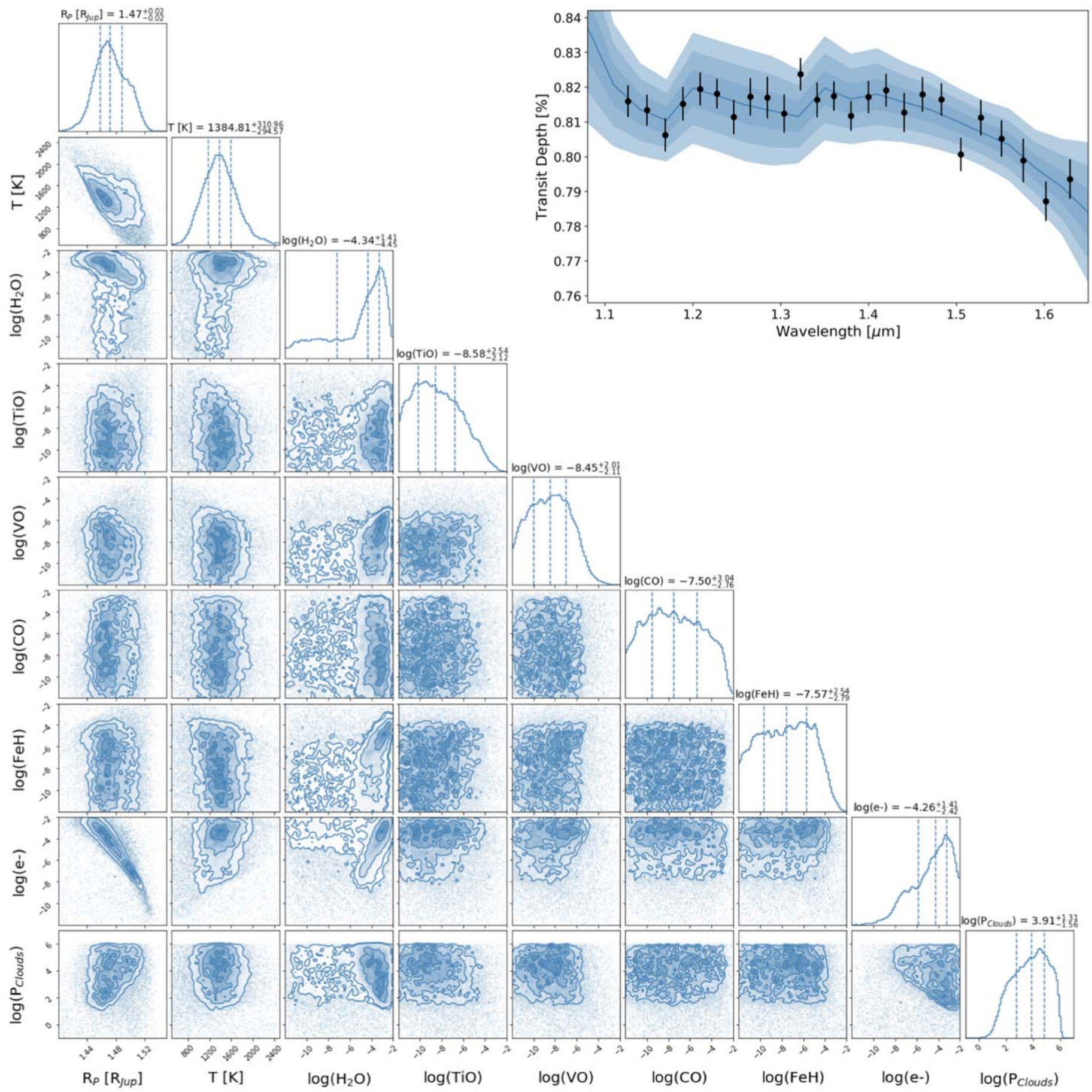

Figure 3. KELT-7 b atmospheric retrieval posterior distributions of the transmission spectrum and the best-fit model with $3 \sigma$ confidence in blue while the HST WFC 3 data is represented by the black data points.

presence of water and dissociated hydrogen, as shown by the posterior distributions in Figure 3. We calculate the Atmospheric Detectability Index (ADI; Tsiaras et al. 2018) to be 16.8 for the transmission spectrum, indicating strong evidence of atmospheric features. The retrieved temperature of around 1400 $\mathrm{K}$ for the terminator region is consistent with the expected value given the equilibrium temperature. However, as we are in a temperature regime $\left(T_{\mathrm{eq}} \simeq 2000 \mathrm{~K}\right)$ where $3 \mathrm{D}$ effects across the limb could occur, we may be biased on the abundances and temperature retrieved (Pluriel et al. 2020).

In contrast, the extracted emission spectrum does not contain strong absorption or emission features. Although the data is not consistent with a simple blackbody, as shown in Figure 4, it can be explained by a varying temperaturepressure profile and $\mathrm{H}^{-}$. We calculate the ADI against a simple blackbody to be 19.6, highlighting the poorness of the 


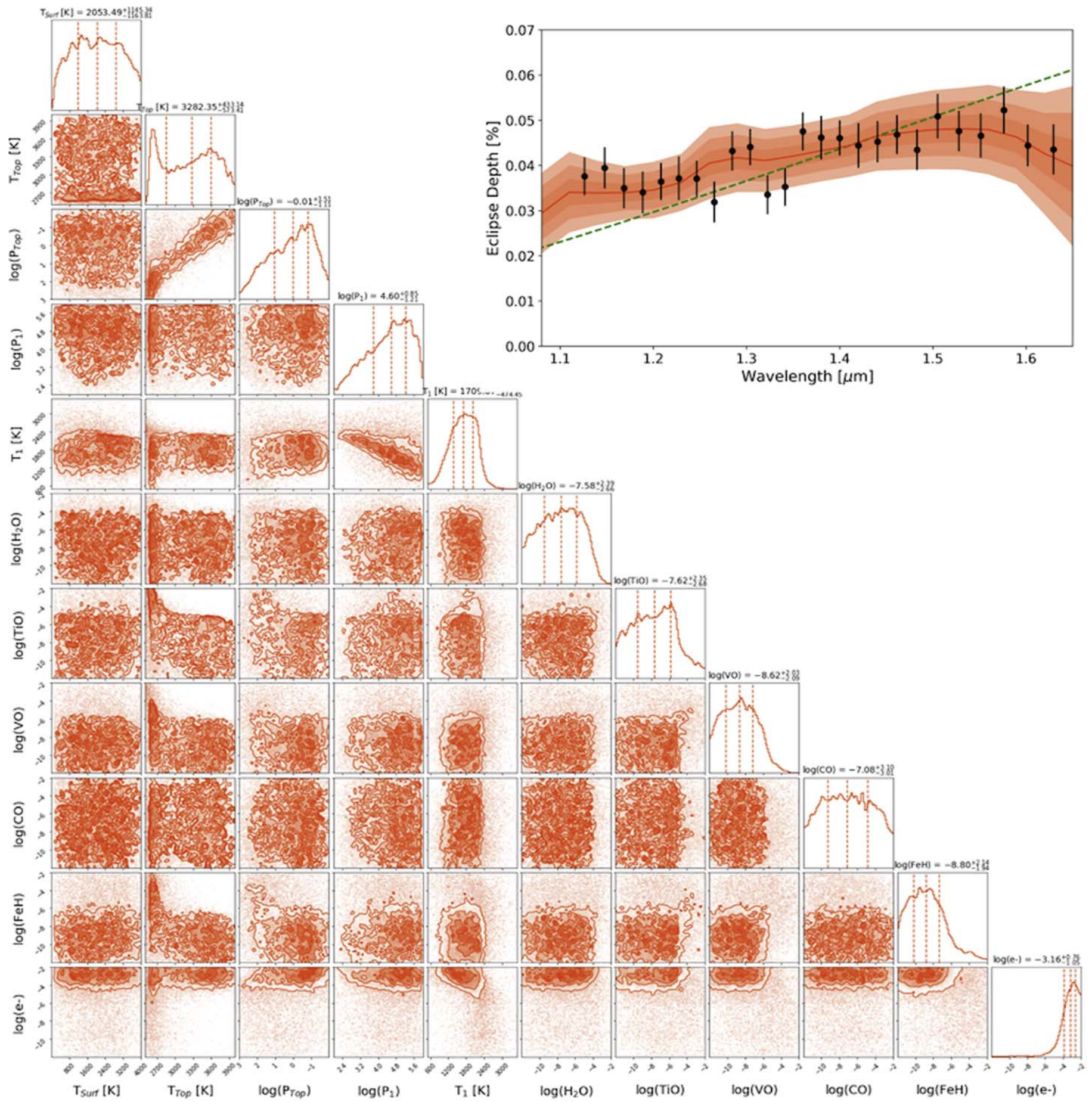

Figure 4. KELT-7 b atmospheric retrieval posterior distributions of the emission spectrum and the best fit retrieved with $3 \sigma$ confidence in red while the HST WFC 3 data is represented by the black data points. Also shown is a blackbody fit (green) that converged to a temperature of $T_{\mathrm{BB}} \simeq 2500 \mathrm{~K}$ and does not well describe the observations.

blackbody fit and indicating the presence of a detectable atmosphere. However, it could also indicate that 2D effects, such as those suggested by Wilkins et al. (2014), are affecting the observed spectrum. In their study of CoRoT-2 $b$ they observed an emission spectrum similar to that of KELT-7 $b$ (i.e., one that is poorly fit by a blackbody but that can be better fit using two blackbodies). Such inhomogeneities will certainly be important in the analysis of emission data from future missions (Taylor et al. 2020). Our best fit favors a thermal inversion in the stratosphere of KELT-7 b. Although the lower part of the atmosphere has large temperature uncertainties (between 1000 and $3000 \mathrm{~K}$ ), the middle and top temperature-pressure points are well constrained enough to indicate a thermal inversion with a temperature at the top 

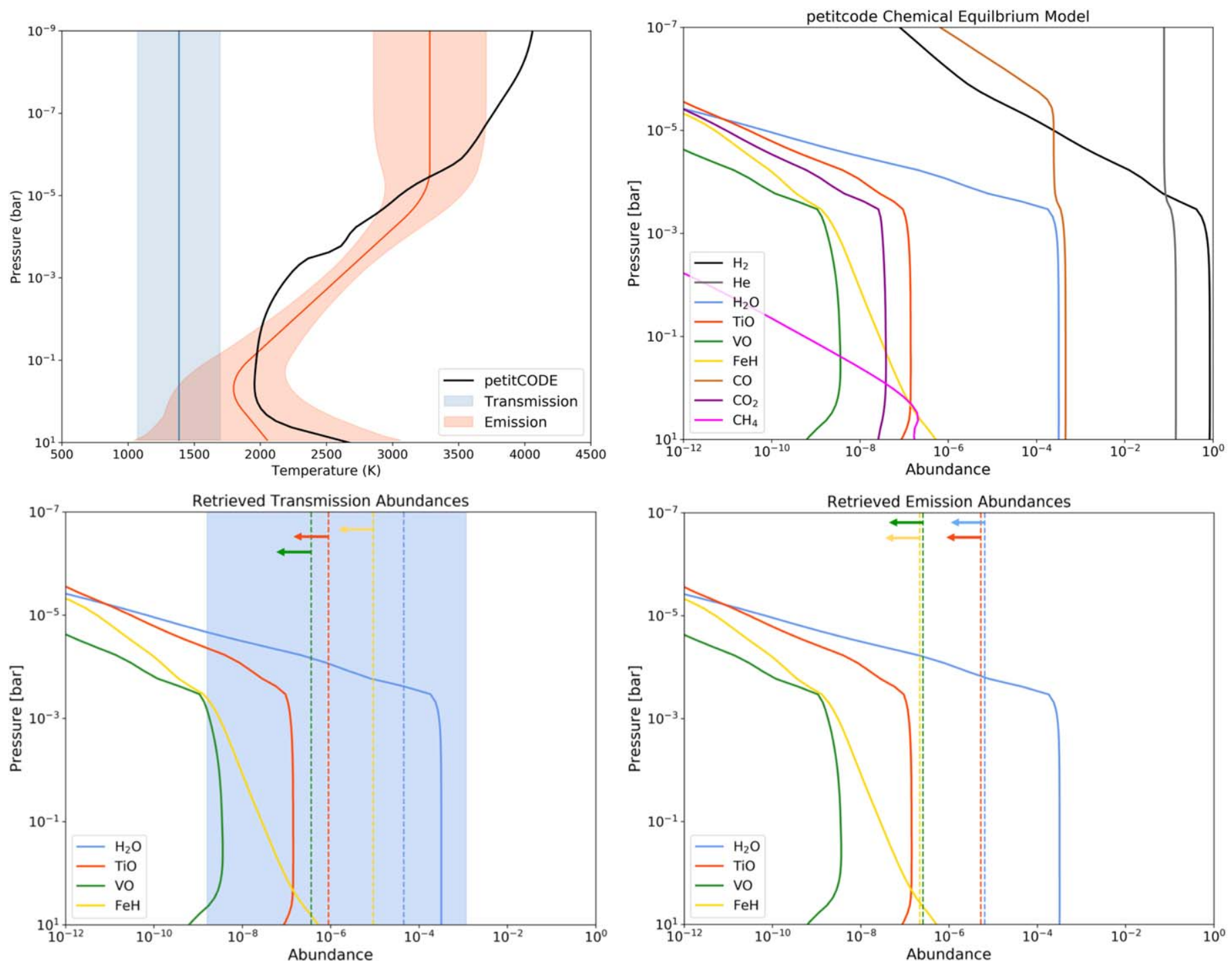

Figure 5. Results of our self-consistent petitCODE model for KELT-7 b and our retrievals on WFC 3 data. Top left: comparison of the temperature-pressure profiles. The petitCODE model (black) features a thermal inversion at 1 mbar, because of absorption by TiO and VO, and closely matches the retrieved profile. Top right: molecular abundances for the petitCODE simulation. The equilibrium fractions of most molecules remain approximately constant for pressures higher than 1 mbar, but drop quickly at lower atmospheric pressures due to thermal dissociation. Bottom left: comparison of constrained molecular abundances in transmission (dotted lines) to those from the petitCODE simulation (solid lines). The water abundance is seen to be within $1 \sigma$ of that which is predicted. Bottom right: comparison of upper bounds placed on molecular abundances in emission to those from the petitCODE simulation. The $1 \sigma$ upper bound on the water abundance is significantly lower than the petitCODE simulations. In both transmission and emission, the $1 \sigma$ upper bounds on $\mathrm{TiO}, \mathrm{VO}$, and $\mathrm{FeH}$ are well above the predicted abundances, suggesting that the data is not sufficient to comment on their presence/absence.

between 2900 and $3700 \mathrm{~K}$ as shown in Figure 5. Both transmission and emission spectra, along with their best-fit solutions are shown in Figures 3 and 4.

The retrieved emission temperature-pressure profile closely agrees with the petitCODE simulations, both showing a temperature inversion as shown in Figure 5. The retrieved water abundance in transmission is consistent with the predictions although the $1 \sigma$ upper bound in emission is below what is expected. For $\mathrm{TiO}$, $\mathrm{VO}$, and $\mathrm{FeH}$, the upper bound place on their abundances is significantly above the amount expected from our petitCODE simulations, suggesting the HST WFC3 data is no sensitive enough for us to conclude on their presence/absence.

The transits of KELT-7 $b$ from HST and TESS were seen to arrive early compared to the predictions from Bieryla et al. (2015). As the observation did not include ingress or egress, the
HST transit fitting is not as precise, and potentially not as accurate, as the TESS data which captured the whole event. Using this new data, we determined the ephemeris of KELT-7 $\mathrm{b}$ to be $P=2.73476541 \pm 0.00000036$ days and $T_{0}=2458384.425577 \pm 0.000099 \mathrm{BJD}_{\mathrm{TDB}}$ where $P$ is the planet's period, $T_{0}$ is the reference mid-time of the transit, and $\mathrm{BJD}_{\mathrm{TDB}}$ is the barycentric Julian date in the barycentric dynamical. Our derived period is $0.84 \mathrm{~s}$ and $0.063 \mathrm{~s}$ shorter than the periods from Bieryla et al. (2015) and Garhart et al. (2020) respectively. The $\sim 10$ minute residual of the TESS transits from the ephemeris of Bieryla et al. (2015) show the necessity to regularly follow-up transiting planets and for programs such as ExoClock ${ }^{19}$ to which our observations have been uploaded. By the launch of the Atmospheric

\footnotetext{
${ }^{19}$ https://www.exoclock.space
} 

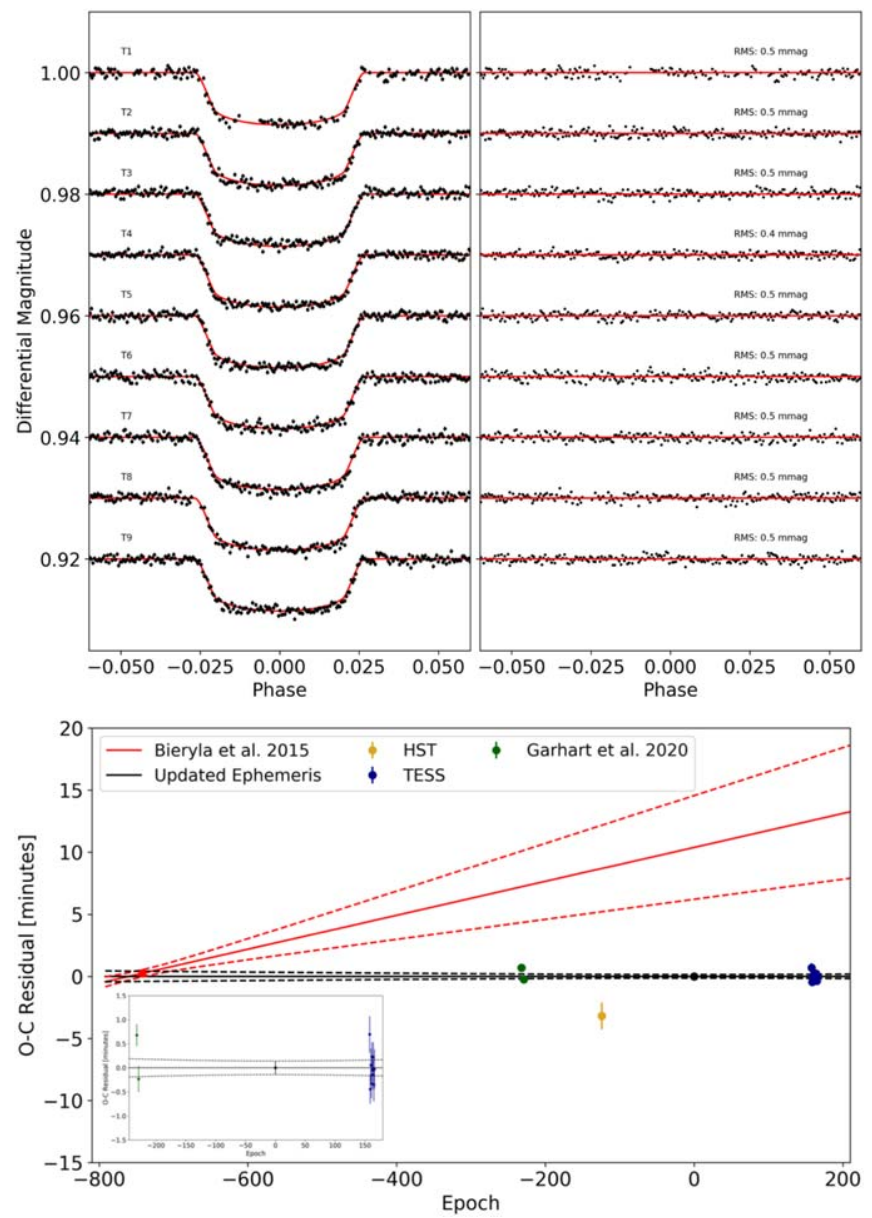

Figure 6. Top: TESS observations of KELT-7 b presented in this work. Left: detrended data and best-fit model. Right: residuals from fitting. Bottom: observed minus calculated $(O-C)$ mid-transit times for KELT-7 b. Transit mid-time measurements from this work are shown in gold (HST) and blue (TESS), while the literature $T_{0}$ value is in red. The black line denotes the new ephemeris of this work with the dashed lines showing the associated $1 \sigma$ uncertainties and the black plot data point indicating the updated $T_{0}$. For comparison, the previous literature ephemeris and their $1 \sigma$ uncertainties are given in red. The inset figure shows a zoomed plot that highlights the precision of the TESS mid-time fits.

Table 3

Transit Mid-times Used to Refine the Ephemeris of Planets from This Study

\begin{tabular}{lcc}
\hline \hline Epoch & Transit Mid-time $\left(\mathrm{BJD}_{\mathrm{TDB}}\right)$ & References \\
\hline-742 & $2456355.229809 \pm 0.000198$ & Bieryla et al. (2015) \\
-232 & $2457749.959530 \pm 0.000160$ & Garhart et al. (2020) \\
-229 & $2457758.164460 \pm 0.000190$ & Garhart et al. (2020) $^{\mathrm{a}}$ \\
-124 & $2458045.316888 \pm 0.000627$ & This work \\
158 & $2458816.518025 \pm 0.000282$ & This work \\
159 & $2458819.253548 \pm 0.000220$ & This work \\
160 & $2458821.987982 \pm 0.000202$ & This work \\
161 & $2458824.723075 \pm 0.000183$ & This work \\
162 & $2458827.457521 \pm 0.000214$ & This work \\
163 & $2458830.192334 \pm 0.000196$ & This work \\
164 & $2458832.927100 \pm 0.000212$ & This work \\
165 & $2458835.661872 \pm 0.000246$ & This work \\
166 & $2458838.396646 \pm 0.000194$ & This work \\
\hline
\end{tabular}

Notes. All mid-times reported in this work are from TESS unless otherwise stated.

a Data from Hubble.
Remote-sensing Infrared Exoplanet Large-survey (ARIEL), in late 2028, around 1400 orbits of KELT-7 $b$ will have occurred since the $T_{0}$ derived here and the difference in the predicted transit times between the ephemeris from this work and from Garhart et al. (2020) would be around 5 minutes. The observed minus calculated residuals, along with the fitted TESS light curves are shown in Figure 6 while the fitted mid-times can be found in Table 3.

\section{Discussion}

In transmission, $\mathrm{H}_{2} \mathrm{O}$ and $\mathrm{H}^{-},\left(\log \left(\mathrm{H}_{2} \mathrm{O}\right)=-4.34_{-4.45}^{+1.41}\right.$ and $\left.\log \left(\mathrm{H}^{-}\right)=-4.26_{-2.42}^{+1.41}\right)$ are well defined. As shown in the posterior distributions in Figure 3, correlations exist between the abundances of the molecules, particularly between $\mathrm{H}_{2} \mathrm{O}$ and $\mathrm{H}^{-}$. We could expect to also find $\mathrm{CO}$ in such a hot atmosphere ( $T_{\text {eq }} \simeq 2000 \mathrm{~K}$ ), evidence for which has been seen for other hot Jupiters: WASP-121b (Evans et al. 2017; Parmentier et al. 2018) and WASP-33b (Haynes et al. 2015). However our retrieval analysis on HST data only provides no evidence for the presence of these molecules. We also explored the addition of Spitzer/IRAC and TESS data, see Section 4.1 for more details.

The nondetection of $\mathrm{TiO}$ and $\mathrm{VO}$ in the terminator region agreed with predictions from Spiegel et al. (2009). Their work suggests that in highly irradiated atmospheres, similar to KELT-7 b, TiO and VO would be likely to rain out in cold traps and disappear from the visible atmosphere. Observational evidence for these processes was reported in the emission spectrum of Kepler-13Ab (Beatty et al. (2017). To overcome these effects, large advective mixing and higher temperatures (higher than $1800 \mathrm{~K}$ ) would be required, leading to a large abundance of $\mathrm{VO}$ in the dayside of the planet. However, in our analysis, we do not find evidence for VO or $\mathrm{TiO}$ on the dayside of KELT-7 $\mathrm{b}$, which could be unraveled with higher $\mathrm{S} / \mathrm{N}$ in future observations such as ARIEL or the James Webb Space Telescope (JWST). Also, our analysis potentially finds a large difference in the day/night temperatures with thermal inversion on the dayside, which would indicate that day- and nightside of the planet are poorly coupled by large-scale dynamical processes, thus preventing $\mathrm{VO}$ and $\mathrm{TiO}$ from reaching the cold nightside and condensating. Fortney et al. (2008) postulated that the presence of optical absorbers would lead, and require, large day-night temperature contrasts, which could suggest that $\mathrm{TiO}$ abd $\mathrm{VO}$ are present but simply beyond the sensitivity of the data.

\subsection{Addition of Spitzer Data}

KELT-7 $\mathrm{b}$ has also been studied, in both transmission and emission, by the Spitzer Space Telescope using each of the 3.6 and $4.5 \mu \mathrm{m}$ channels of the IRAC. Combining data from multiple instruments or observatories has become the standard within the field as a way of increasing the spectral coverage, seeking to break the degeneracies that occur when fitting data over a narrow wavelength range (e.g., Sing et al. 2016). However, such a procedure is fraught with risk due to potential incompatibilities between data sets. First, it has been shown using different orbital parameters $(a / R s$ and $i)$ in the fitting of the data can lead to offsets between the data sets (Yip et al. 2019). Second, the choice of limb-darkening coefficients can cause vertical shifts in the spectrum 
Table 4

Spectral Data of KELT-7 b Used in This Study

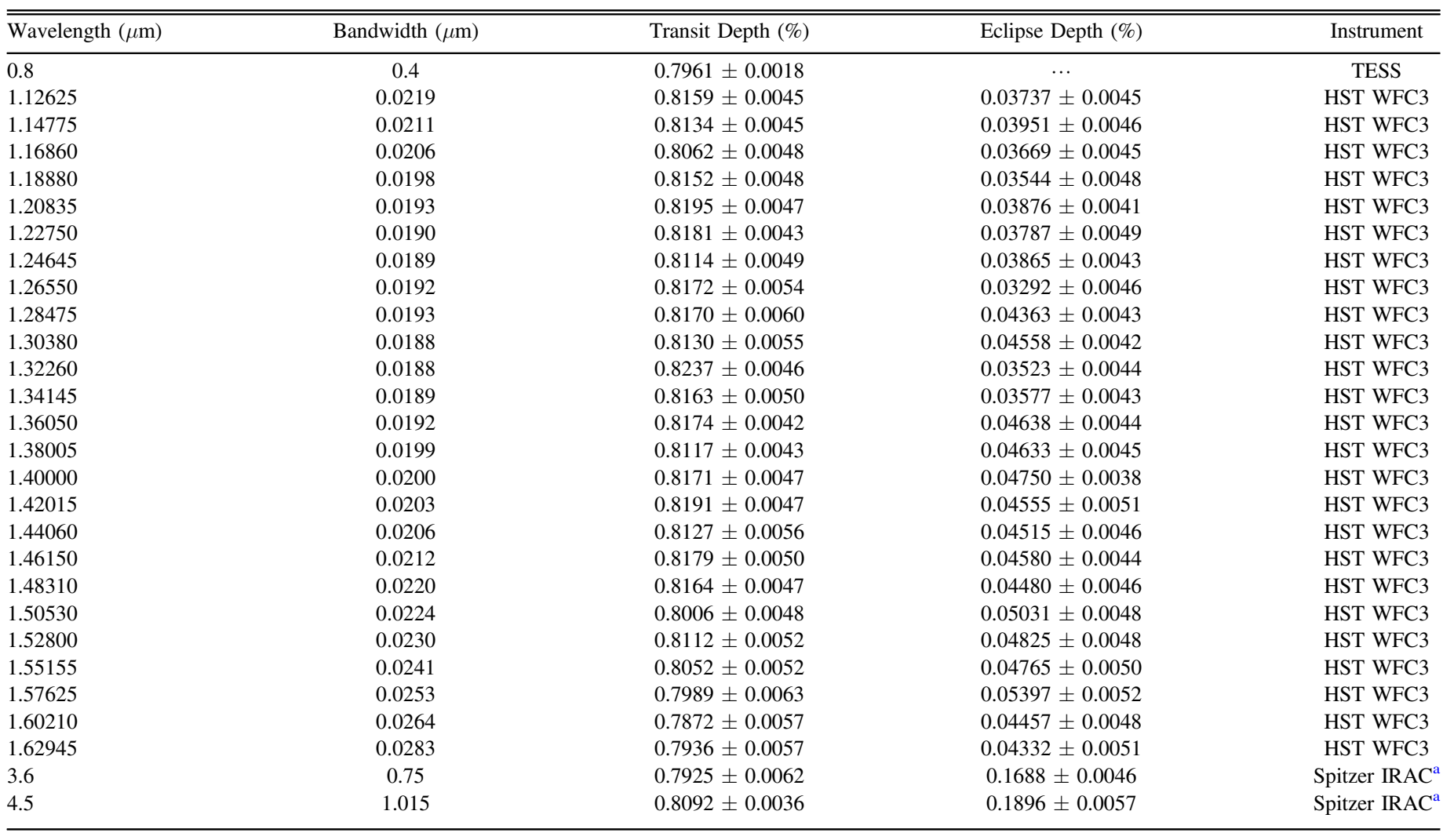

Note.

' Taken from Garhart et al. (2020).

(Tsiaras et al. 2018). Third, stellar variability, activity, or spots can also induce offsets in the recovered spectra (e.g., Bruno et al. 2020). Finally, imperfect correction of instrument systematics can alter the fitted white light-curve depth, again generating shifts between data sets, making the derived transit/eclipse depths incompatible (e.g., Diamond-Lowe et al. 2014; Stevenson et al. 2014a, 2014b). Each of these effects can of course affect HST data alone but, in this case, the offset would likely only lead to slight changes in the retrieved temperature or radius. When combining data sets, for instance HST WFC3 and Spitzer IRAC, offsets in one of these, or differing offsets in both, could lead to wrongly recovered abundances. In transmission and emission, Spitzer observations are sensitive to $\mathrm{CH}_{4}$ in the $3.6 \mu \mathrm{m}$ band and $\mathrm{CO}$ or $\mathrm{CO}_{2}$ in the $4.5 \mu \mathrm{m}$ band while WFC 3 data cannot constrain these molecules. Thus the detection, or nondetection, would be based entirely off the two Spitzer bands relative to the WFC3 data. An offset in either one of these would instigate the incorrect recovery of the $\mathrm{CH}_{4}, \mathrm{CO}$, and $\mathrm{CO}_{2}$ abundances. In emission, these Spitzer points have been used as evidence for the presence of, or lack of, a thermal inversion. Differences in the correction of systematics have led to discrepant results (e.g., HD 209458b; Knutson et al. 2008; Diamond-Lowe et al. 2014).

Here, we tentatively add the Spitzer data for KELT-7 b, taken from the study by Garhart et al. (2020) of 36 hot Jupiters. For the fitting of the Spitzer eclipses, Garhart et al. (2020) froze the orbital parameters to those from Bieryla et al. (2015), overcoming the first hurdle about combining data sets. The transit observations were also fitted with fixed orbital parameters and limb-darkening coefficients from Claret (2000), again showing consistency with our data. The latter two issues, of stellar variability or activity and the detrending of instrument systematics, cannot be easily determined without an overlap in spectral coverage. Therefore, we caution the reader that the compatibility of the data sets cannot be guaranteed. Additionally, we cautiously added the TESS data which was again fitted with same orbital parameters and limb-darkening laws.

The best-fit retrieved spectra, both with and without the Spitzer data, is shown in Figure 7. Little difference is seen between the fits, particularly in transmission where the best fits are almost constantly within $1 \sigma$ of each other. The recovered temperature-pressure profiles are also practically identical. While this may suggest that the data is compatible, it also shows that the information content of adding Spitzer, in this case, is relatively low. Therefore this begs the question of whether risking data incompatibility is worthwhile when there is little to gain. Figures 8 and 9 show posteriors from the fittings with and without Spitzer, again highlighting the similarity between the fits. In transmission the only noticeable difference is in the recovered $\mathrm{CO}$ abundance, which is not constrained in the case of HST alone but the addition of Spitzer suggests an abundance of $\log (\mathrm{CO})=$ $-4.56_{-4.69}^{+1.72}$. The second change is in the water abundance 

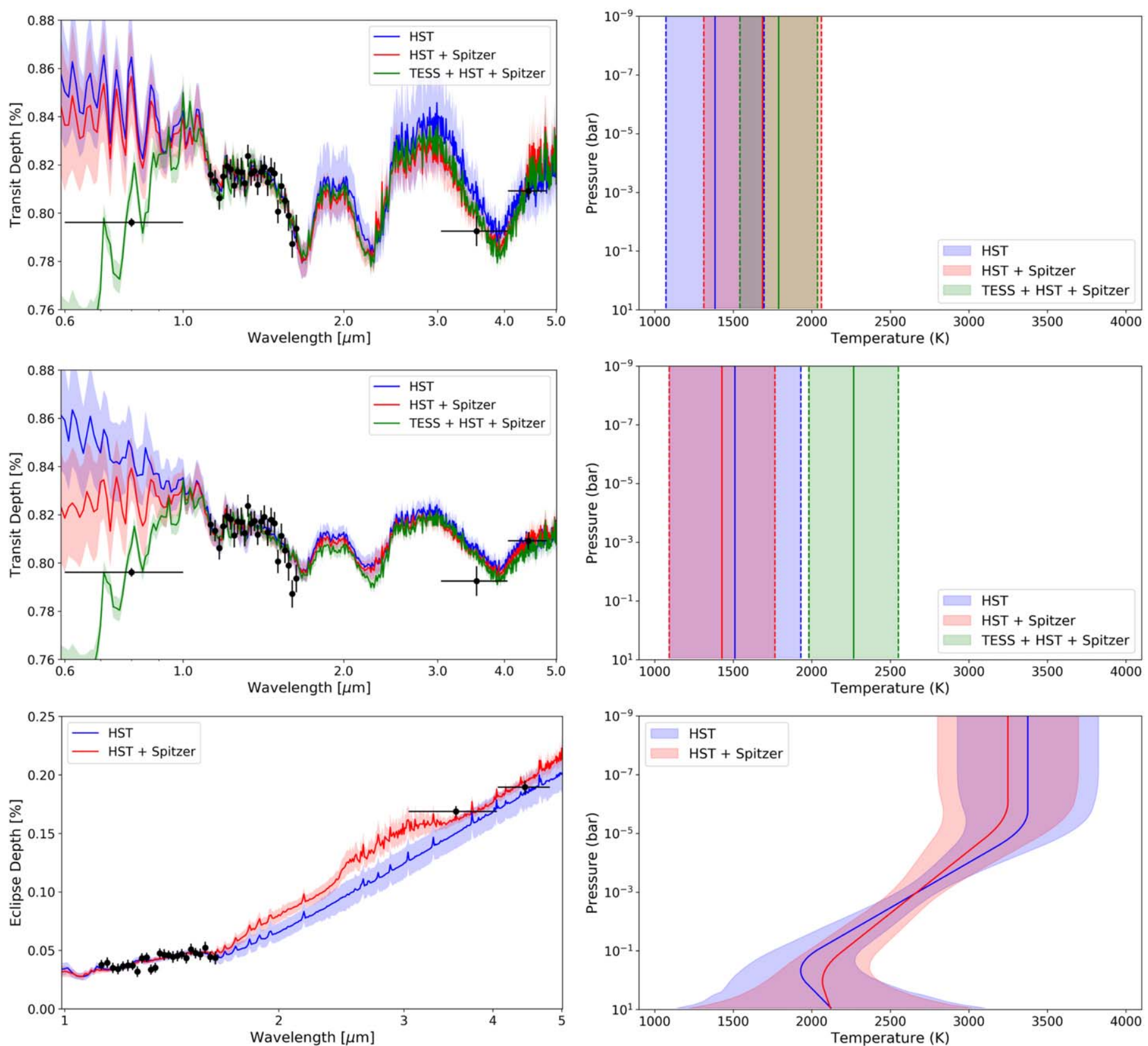

Figure 7. Best-fit spectra (left) and temperature-pressure profiles (right), with $1 \sigma$ errors in each case, for transit (top and middle) and eclipse (bottom) observations of KELT-7 b with HST data only, HST and Spitzer (red), and HST, Spitzer, and TESS (green). The top transmission plots, and the emission case, include the $\mathrm{H}^{-}$opacity while the middle plots do not.

recovered in emission, with HST and Spitzer converging to $\log \left(\mathrm{H}_{2} \mathrm{O}\right)=-5.11_{-4.03}^{+1.37}$ while no constraint can be made in the HST-only case.

On the other hand, the addition of the TESS transmission data drastically changes the solution, removing the detection of dissociated hydrogen, instead preferring $\mathrm{FeH}$ to explain the absorption at the shorter wavelengths within the G141 grism due to the shallow TESS transit depth. We also explored the retrieved atmospheric abundances in transmission without the $\mathrm{H}^{-}$opacity, again finding that there is little difference when adding Spitzer data as shown in Figure 10. In this case, all data combinations readily agree on the abundances of $\mathrm{H}_{2} \mathrm{O}, \mathrm{FeH}$, and $\mathrm{CO}$. However, differences are seen in the evidence for $\mathrm{TiO}$ and $\mathrm{VO}$, the presence of which the addition of TESS data of rules out for $\log (\mathrm{TiO}, \mathrm{VO})>-10$, and in the recovered radius and terminator temperature, with the TESS data set preferring a lower radius and higher temperature. We suggest it is imperative to, at the very least, study multi-instrument data sets separately, as well as combined, when doing model fitting, as we have done in this study.

\subsection{Future Characterization}

The most effective solution to understanding the source of the absorption seen in transmission between 1.1 and $1.3 \mu \mathrm{m}$ would be to take more data, namely with the G102 grism of WFC3, which covers $0.8-1.1 \mu \mathrm{m}$. As there is also archival 


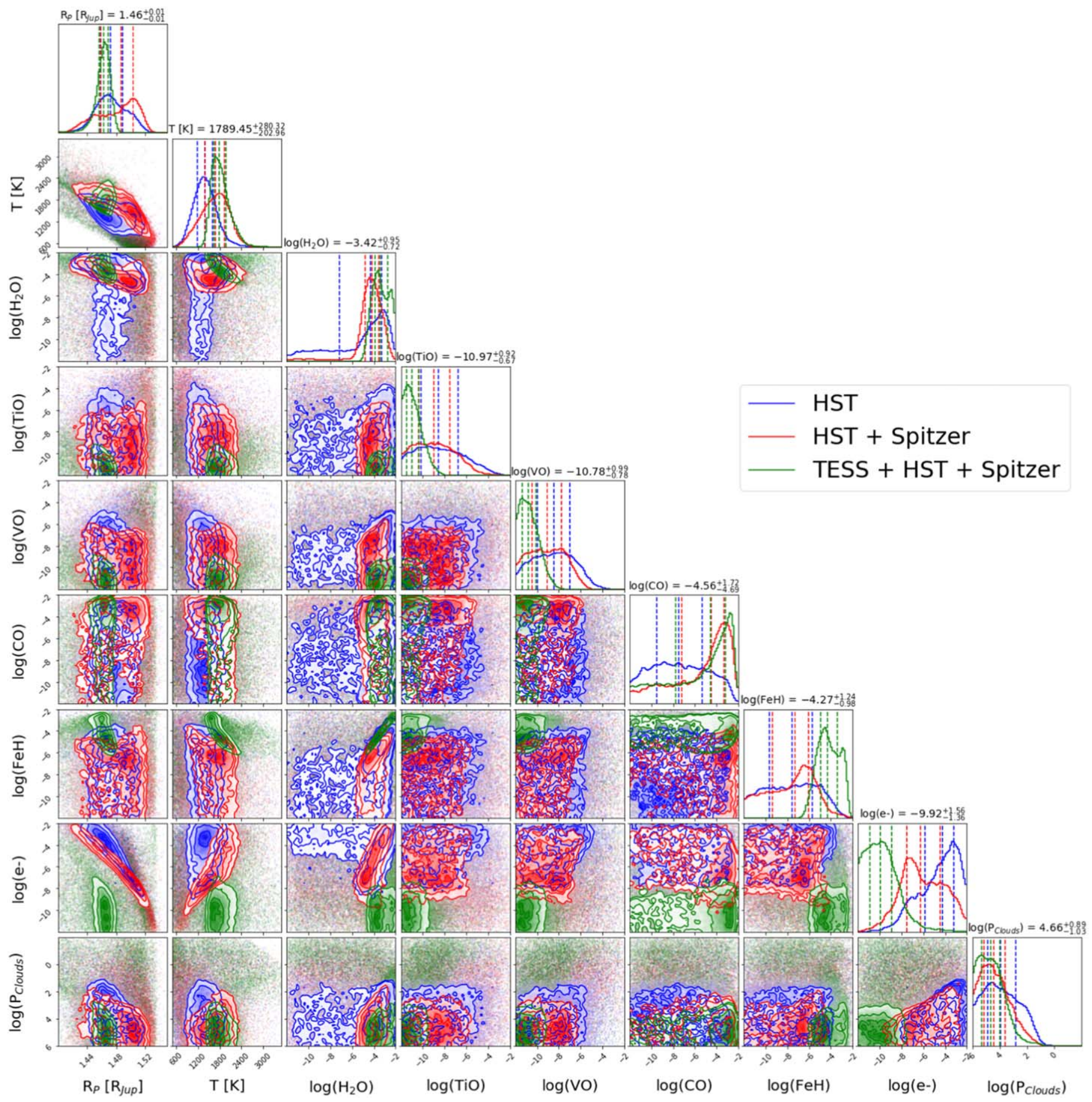

Figure 8. Posterior distributions for atmospheric retrievals of KELT-7 b with various data sets. The addition of Spitzer data brings little change to the atmospheric properties while TESS data drives the retrieval to favor $\mathrm{FeH}$ over $\mathrm{H}^{-}$. We note that this $\mathrm{FeH}$ abundance is far above the expected (log(FeH) $\sim-7$ ). The reported values for each parameter are those obtained with the fit on all three data sets.

Space Telescope Imaging Spectrograph (STIS) transmission data for KELT-7 b, with the G430L and G750L grisms, this would provide continuous coverage from 0.3 to $1.6 \mu \mathrm{m}$, allowing for better constraints on the abundances of these optical absorbers. We would also advocate for additional eclipse observations with WFC3, with either grism, to increase the spectral coverage and/or increase the $\mathrm{S} / \mathrm{N}$, which may allow for spectral features to be uncovered.

Additionally, future space telescopes JWST (Greene et al. 2016), Twinkle (Edwards et al. 2018), and ARIEL (Tinetti et al. 2018) will provide a far wider wavelength range and these missions will definitively move the exoplanet field from an era 


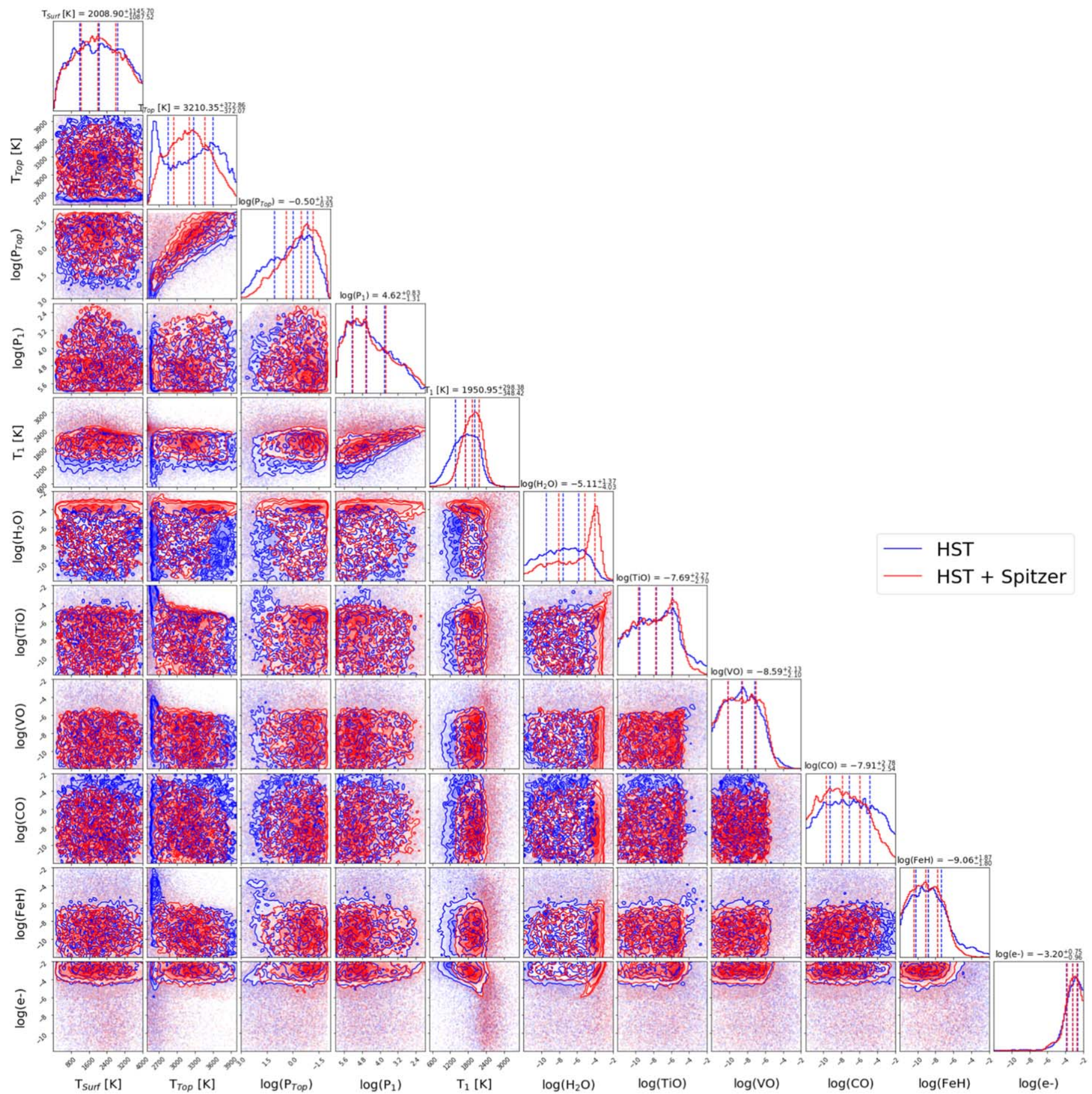

Figure 9. Posterior distributions for atmospheric retrievals of the emission spectra of KELT-7 b with and without Spitzer data (blue and red respectively). The addition of Spitzer data brings little change to the atmospheric properties except the derived water abundance which appears clearly with Spitzer data. The reported values for each parameter are those obtained with the fit on all three data sets.

of detection into one of characterization, allowing for the identification of the molecular species present and their chemical profile, insights into the atmospheric temperature profile, and the detection and characterization of clouds. ARIEL, the ESA M4 mission due for launch in 2028, will conduct a survey of $\sim 1000$ planets to answer the question: how chemically diverse are the atmospheres of exoplanets? KELT-7 b has been identified as an excellent target for study with ARIEL (Edwards et al. 2019), through both transmission and emission spectroscopy, and simulated error bars from Mugnai et al. (2020) have been added to the best-fit spectra to showcase this. Figure 11 shows simulated ARIEL and JWST observations and highlights the wavelength coverage performed by those future missions. Additionally ExoWebb (B. Edwards et al. 2020, in preparation) has been used to showcase the capability of JWST for studying this planet. 


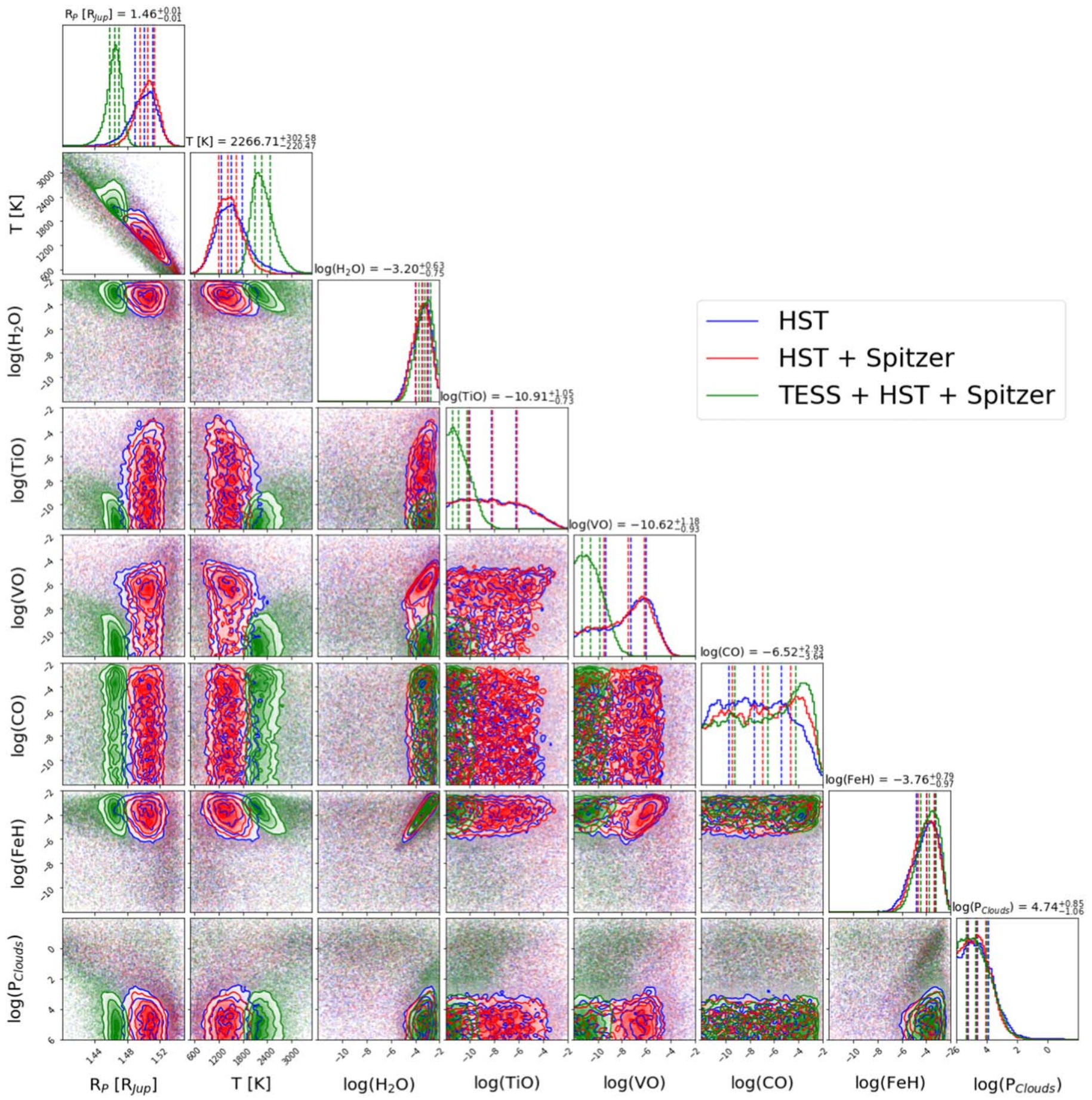

Figure 10. Posterior distributions for atmospheric retrievals of the transmission spectra of KELT-7 $\mathrm{b}$ with various data sets, this time without including $\mathrm{H}^{-}$as an opacity source. The addition of Spitzer data again has little effect on the retrieved atmospheric properties while TESS data drives the retrieval to higher temperatures and rules out the presence of $\mathrm{TiO}$ and $\mathrm{VO}$. All cases require an $\mathrm{FeH}$ abundance that is far greater than expected. The reported values for each parameter are those obtained with the fit on all three data sets.

\section{Conclusion}

We present spectroscopic transmission and emission observations of KELT-7 b taken with Hubble WFC3. While the transit spectra demonstrates strong absorption features indicative of $\mathrm{H}_{2} \mathrm{O}$ and $\mathrm{H}^{-}$, the emission spectrum lacks features and can be fitted with CIA alone. We also explore adding data from Spitzer IRAC, with the results being very similar in both transmission and emission. Finally, we find that adding TESS data in our analysis strongly modifies our results. As these instruments do not provide spectral overlap, more data is needed to fully understand the source of the optical absorption seen in transmission. Further observations with Hubble, or with the next generation of observatories, will undoubtedly allow for an enhanced probing of the atmosphere of this intriguing planet. The analysis of archival Hubble data is an essential preparatory step in enriching our comprehension of exoplanet 

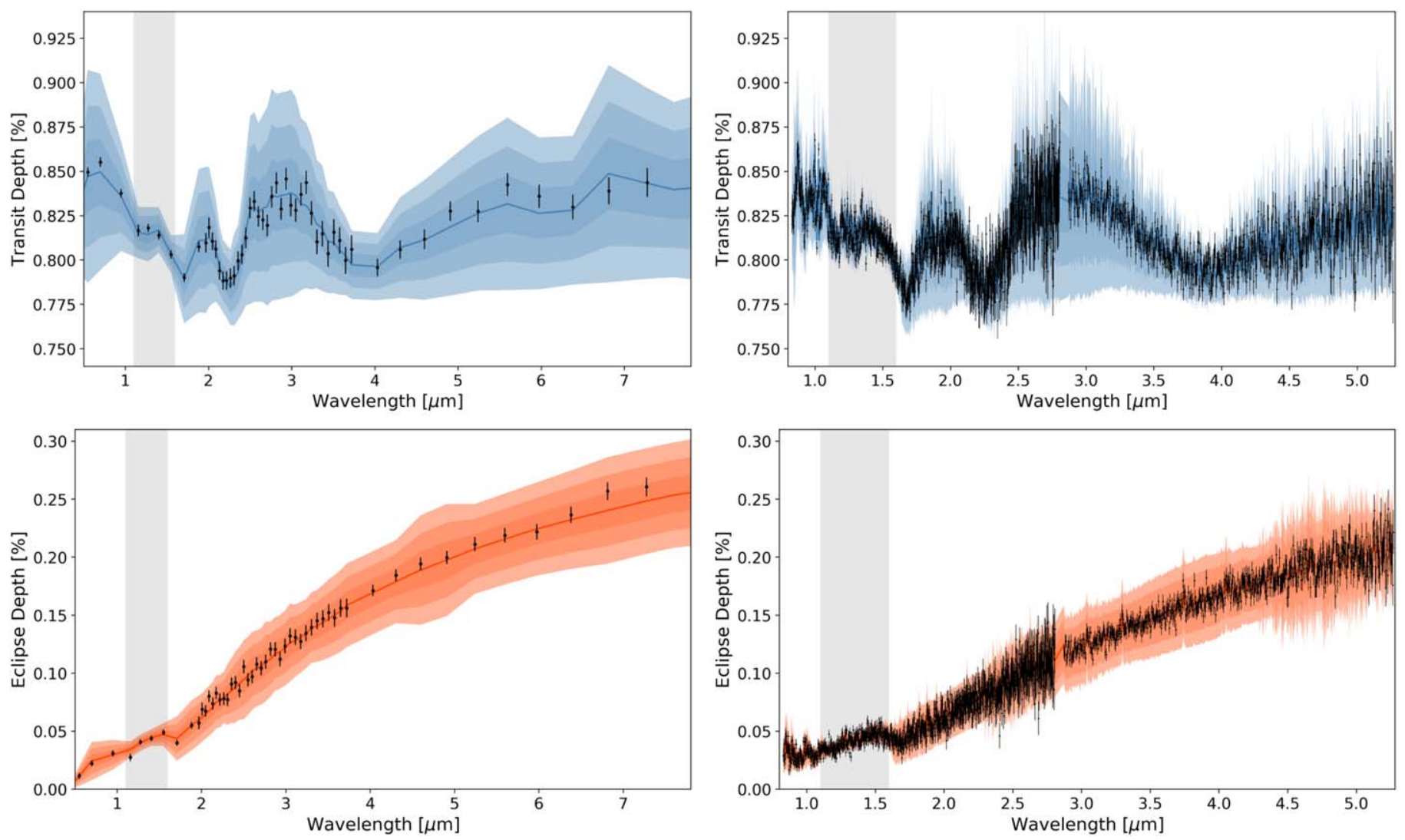

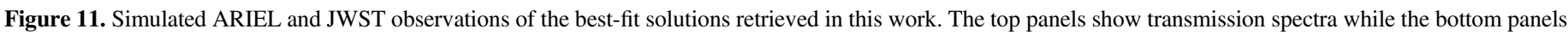

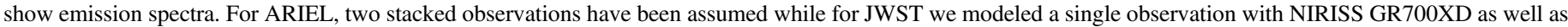
an observation with NIRSpec G395M.

atmospheres, allowing us to begin to appreciate their true diversity and understand the optimal observation strategy for upcoming facilities.

This work was realized as part of ARES, the Ariel Retrieval Exoplanet School, in Biarritz in 2019. The school was organized by Jean-Philippe Beaulieu, Angelos Tsiaras, and Ingo Waldmann with the financial support of CNES. This work is based upon observations with the NASA/ESA Hubble Space Telescope, obtained at the Space Telescope Science Institute (STScI) operated by AURA, Inc. The publicly available HST observations presented here were taken as part of proposal 14767, led by David Sing. These were obtained from the Hubble Archive which is part of the Mikulski Archive for Space Telescopes. This paper includes data collected by the TESS mission which is funded by the NASA Explorer Program. TESS data is publicly available via the Mikulski Archive for Space Telescopes (MAST). This work is also based in part on observations made with the Spitzer Space Telescope, which is operated by the Jet Propulsion Laboratory, California Institute of Technology, under a contract with NASA. J.P.B. acknowledges the support of the University of Tasmania through the UTAS Foundation and the endowed Warren Chair in Astronomy, Rodolphe Cledassou, Pascale Danto, and Michel Viso (CNES). B.E., Q.C., M.M., A.T., and I.W. are funded through the ERC Starter Grant ExoAI (GA 758892) and the STFC grants ST/P000282/1, ST/P002153/1, ST/ S002634/1, and ST/T001836/1. N.S. acknowledges the support of the IRIS-OCAV, PSL. M.P. acknowledges support by the European Research Council under Grant Agreement
ATMO 757858 and by the CNES. R.B. is a PhD fellow of the Research Foundation-Flanders (FWO). W.P. and T.Z. have received funding from the European Research Council (ERC) under the European Union's Horizon 2020 research and innovation program (grant agreement No. 679030/WHIPLASH). O.V. thanks the CNRS/INSU Programme National de Planétologie (PNP) and CNES for funding support. G.G. acknowledges the financial support of the $2017 \mathrm{PhD}$ fellowship program of INAF. L.V.M. and D.M.G. acknowledge the financial support of the ARIEL ASI grant No. 2018-22-HH.0.

Software: Iraclis (Tsiaras et al. 2016b), TauREx3 (A. F. AlRefaie et al. 2019, in preparation), pylightcurve (Tsiaras et al. 2016a), ExoTETHyS (Morello et al. 2020), ArielRad (Mugnai et al. 2020), ExoWebb (B. Edwards et al. 2020, in preparation), Astropy (Astropy Collaboration et al. 2018), h5py (Collette 2013), emcee (Foreman-Mackey et al. 2013), Matplotlib (Hunter 2007), Multinest (Feroz et al. 2009), Pandas (McKinney 2011), Numpy (Oliphant 2006), SciPy (Virtanen et al. 2020).

\section{Appendix}

TauREx3 employs Bayesian statistics as the cornerstone for the retrieval analysis (Waldmann et al. 2015a, 2015b). Bayes' theorem states that

$$
P(\theta \mid x, \mathcal{M})=\frac{P(x \mid \theta, \mathcal{M}) P(\theta, \mathcal{M})}{P(x \mid \mathcal{M})},
$$

where $P(\theta, \mathcal{M})$ is the Bayesian prior and $\mathcal{M}$ is the forward model. $P(\theta \mid x, \mathcal{M})$ is the posterior probability of the model 
parameters $\theta$ given the data, $x$ assuming the forward model $\mathcal{M}$. A Bayesian analysis is implemented in TauREx3 via nested sampling (NS).

TauREx3 includes the implementation of NS Bayesian statistics via Multinest (Feroz \& Hobson 2008; Feroz et al. 2009, 2019). NS employs a Monte Carlo approach that constrains via ellipsoids encompassing the parameter space of the highest likelihood. NS determines the Bayesian evidence which is given by

$$
E=\int P(\theta \mid \mathcal{M}) P(x \mid \theta, \mathcal{M}) d \theta
$$

where $P(x, \mathcal{M})$ is the evidence. These statistical products produced by Multinest are used to perform the best-fit model selection. NS performed by Multinest also allows for efficient parallelization which permits the usage of high performance cluster computing.

\section{ORCID iDs}

Billy Edwards (iD https://orcid.org/0000-0002-5494-3237 Quentin Changeat (iD https://orcid.org/0000-0001-6516-4493 Ahmed Al-Refaie (i) https://orcid.org/0000-0003-2241-5330 Mario Morvan (iD https://orcid.org/0000-0001-8587-2112 Lorenzo V. Mugnai (ib https://orcid.org/0000-0002-9007-9802 Nour Skaf (iD https://orcid.org/0000-0002-9372-5056 Tiziano Zingales (i) https://orcid.org/0000-0001-6880-5356 Benjamin Charnay (iD https://orcid.org/0000-0003-0977-6545 Jérémy Leconte (i) https://orcid.org/0000-0002-3555-480X Angelos Tsiaras (1) https://orcid.org/0000-0003-3840-1793 Olivia Venot (1D https://orcid.org/0000-0003-2854-765X Ingo Waldmann (ib https://orcid.org/0000-0002-4205-5267 Jean-Philippe Beaulieu (iD https://orcid.org/0000-0003-0014-3354

\section{References}

Abel, M., Frommhold, L., Li, X., \& Hunt, K. L. 2011, JPCA, 115, 6805 Abel, M., Frommhold, L., Li, X., \& Hunt, K. L. 2012, JChPh, 136, 044319 Astropy Collaboration, Price-Whelan, A. M., Sipőcz, B. M., et al. 2018, AJ, 156,123

Beatty, T. G., Madhusudhan, N., Tsiaras, A., et al. 2017, AJ, 154, 158

Bieryla, A., Collins, K., Beatty, T. G., et al. 2015, AJ, 150, 12

Bruno, G., Lewis, N. K., Alam, M. K., et al. 2020, MNRAS, 491, 5361

Changeat, Q., Edwards, B., Waldmann, I. P., \& Tinetti, G. 2019, ApJ, 886, 39

Claret, A. 2000, A\&A, 363, 1081

Collette, A. 2013, Python and HDF5 (Sebastopol, CA: OReilly \& Associates)

Deming, D., Wilkins, A., McCullough, P., et al. 2013, ApJ, 774, 95

Diamond-Lowe, H., Stevenson, K. B., Bean, J. L., Line, M. R., \& Fortney, J. J. 2014, ApJ, 796, 66

Dulick, M., Bauschlicher, C. W. J., Burrows, A., et al. 2003, ApJ, 594, 651

Edwards, B., Changeat, Q., Baeyens, R., et al. 2020a, AJ, 160, 8

Edwards, B., Changeat, Q., Yip, K. H., et al. 2020b, MNRAS, in press, arXiv:2005.01684

Edwards, B., Mugnai, L., Tinetti, G., Pascale, E., \& Sarkar, S. 2019, AJ, 157,242

Edwards, B., Rice, M., Zingales, T., et al. 2018, ExA, 47, 29

Espinoza, N., \& Jordán, A. 2015, MNRAS, 450, 1879

Evans, T. M., Sing, D. K., Kataria, T., et al. 2017, Natur, 548, 58

Feroz, F., \& Hobson, M. P. 2008, MNRAS, 384, 449

Feroz, F., Hobson, M. P., \& Bridges, M. 2009, MNRAS, 398, 1601

Feroz, F., Hobson, M. P., Cameron, E., \& Pettitt, A. N. 2019, OJAp, 2, 10

Fletcher, L. N., Gustafsson, M., \& Orton, G. S. 2018, ApJS, 235, 24

Foreman-Mackey, D., Hogg, D. W., Lang, D., \& Goodman, J. 2013, PASP, 125,306
Fortney, J. J., Lodders, K., Marley, M. S., \& Freedman, R. S. 2008, ApJ, 678,1419

Garhart, E., Deming, D., Mandell, A., et al. 2020, AJ, 159, 137

Greene, T. P., Line, M. R., Montero, C., et al. 2016, ApJ, 817, 17

Haynes, K., Mandell, A. M., Madhusudhan, N., Deming, D., \& Knutson, H. 2015, ApJ, 806, 146

Hoeijmakers, H. J., Ehrenreich, D., Kitzmann, D., et al. 2019, A\&A, 627, A165

Hunter, J. D. 2007, CSE, 9, 90

John, T. L. 1988, A\&A, 193, 189

Knutson, H. A., Charbonneau, D., Allen, L. E., Burrows, A., \& Megeath, S. T. 2008, ApJ, 673, 526

Kreidberg, L., Line, M. R., Parmentier, V., et al. 2018, AJ, 156, 17

Kurucz, R. L. 1970, Atlas: a Computer Program for Calculating Model Stellar Atmospheres, SAO Special Report, 309

Li, G., Gordon, I. E., Rothman, L. S., et al. 2015, ApJS, 216, 15

MacDonald, R. J., \& Madhusudhan, N. 2017, MNRAS, 469, 1979

Madhusudhan, N., Knutson, H., Fortney, J. J., \& Barman, T. 2014, in Protostars and Planets VI, ed. H. Beuther, R. Klessen, C. P. Dullemond et al. (Tucson, AZ: Univ. Arizona Press), 739

Martioli, E., Colón, K. D., Angerhausen, D., et al. 2018, MNRAS, 474, 4264

McKemmish, L. K., Masseron, T., Hoeijmakers, H. J., et al. 2019, MNRAS, 488, 2836

McKemmish, L. K., Yurchenko, S. N., \& Tennyson, J. 2016, MNRAS, 463, 771

McKinney, W. 2011, pandas: A Foundational Python Library for Data Analysis and Statistics, https://www.dlr.de/sc/portaldata/15/resources/ dokumente/pyhpc2011/submissions/pyhpc2011_submission_9.pdf

Mikal-Evans, T., Sing, D. K., Goyal, J. M., et al. 2019, MNRAS, 488, 2222

Mollière, P., van Boekel, R., Bouwman, J., et al. 2017, A\&A, 600, A10

Mollière, P., van Boekel, R., Dullemond, C., Henning, T., \& Mordasini, C. 2015, ApJ, 813, 47

Morello, G., Claret, A., Martin-Lagarde, M., et al. 2020, AJ, 159, 75

Mugnai, L. V., Pascale, E., Edwards, B., Papageorgiou, A., \& Sarkar, S. 2020 , ExA, submitted

Oliphant, T. E. 2006, A Guide to NumPy, Vol. 1 (Spanish Fork, UT: Trelgol Publishing)

Parmentier, V., Line, M. R., Bean, J. L., et al. 2018, A\&A, 617, A110

Pluriel, W., Zingales, T., Leconte, J., et al. 2020, A\&A, 636, A66

Polyansky, O. L., Kyuberis, A. A., Zobov, N. F., et al. 2018, MNRAS, 480, 2597

Ricker, G. R., Winn, J. N., Vanderspek, R., et al. 2014, Proc. SPIE, 9143, 914320

Rothman, L. S., Gamache, R. R., Goldman, A., et al. 1987, ApOpt, 26, 4058 Rothman, L. S., Gordon, I. E., Barber, R. J., et al. 2010, JQSRT, 111, 2139

Sing, D. K., Fortney, J. J., Nikolov, N., et al. 2016, Natur, 529, 59

Skaf, N., Fabienne Bieger, M., Edwards, B., et al. 2020, AJ, 160, 109

Spiegel, D. S., Silverio, K., \& Burrows, A. 2009, ApJ, 699, 1487

Stevenson, K. B., Bean, J. L., Fabrycky, D., \& Kreidberg, L. 2014a, ApJ, 796,32

Stevenson, K. B., Bean, J. L., Seifahrt, A., et al. 2014b, AJ, 147, 161

Swain, M. R., Vasisht, G., \& Tinetti, G. 2008, arXiv:0802.1030

Taylor, J., Parmentier, V., Irwin, P. G. J., et al. 2020, MNRAS, 493, 4342

Tennyson, J., Yurchenko, S. N., Al-Refaie, A. F., et al. 2016, JMoSp, 327, 73

Thorngren, D., Gao, P., \& Fortney, J. J. 2019, ApJL, 884, L6

Tinetti, G., Drossart, P., Eccleston, P., et al. 2018, ExA, 46, 135

Tinetti, G., Vidal-Madjar, A., Liang, M.-C., et al. 2007, Natur, 448, 169

Tsiaras, A., Waldmann, I., Rocchetto, M., et al. 2016a, pylightcurve: Exoplanet Lightcurve Model, Astrophysics Source Code Library, ascl:1612.018

Tsiaras, A., Waldmann, I. P., Rocchetto, M., et al. 2016b, ApJ, 832, 202

Tsiaras, A., Waldmann, I. P., Tinetti, G., Tennyson, J., \& Yurchenko, S. N. 2019, NatAs, 3, 1086

Tsiaras, A., Waldmann, I. P., Zingales, T., et al. 2018, AJ, 155, 156 Virtanen, P., Gommers, R., Oliphant, T. E., et al. 2020, NatMe, 17, 261 von Essen, C., Mallonn, M., Welbanks, L., et al. 2019, A\&A, 622, A71 Wakeford, H. R., Sing, D. K., Deming, D., et al. 2013, MNRAS, 435, 3481 Waldmann, I. P., Rocchetto, M., Tinetti, G., et al. 2015a, ApJ, 813, 13 Waldmann, I. P., Tinetti, G., Rocchetto, M., et al. 2015b, ApJ, 802, 107 Wende, S., Reiners, A., Seifahrt, A., \& Bernath, P. F. 2010, A\&A, 523, A58 Wilkins, A. N., Deming, D., Madhusudhan, N., et al. 2014, ApJ, 783, 113 Yip, K. H., Waldmann, I. P., Tsiaras, A., \& Tinetti, G. 2019, arXiv:1811.04686 Zhou, Y., Apai, D., Lew, B. W. P., \& Schneider, G. 2017, AJ, 153, 243 